\section{Transfection of murine dendritic cell line (JAWS II) by a nonviral transfection reagent}

\author{
Shanjana Awasthi and Rebecca A. Cox \\ University of Texas Health Science Center at San Antonio, San Antonio, TX, USA
}

BioTechniques 35:600-604 (September 2003)

Dendritic cells are the most potent antigen-presenting cells that initiate and modulate the host immune system. Based on their immunostimulatory activity, a variety of strategies have been developed to use dendritic cells as vaccines and immunotherapeutic agents against infection and cancer. Genetically modified dendritic cells are useful for immunotherapeutic purposes because of their sustained activity in vivo. However, transfection of dendritic cells with plasmid DNA has been very difficult. While the viral transfection is associated with nonspecific activation of dendritic cells, commonly used nonviral transfection reagents have a low efficiency of transfection. Here we describe an improved, simple, less time-consuming transfection protocol using the nonviral nonliposomal lipid polymer, TransIT-TKO ${ }^{\circledR}$ transfection reagent, for transfecting murine dendritic cells (JAWS II) with the gene that encodes Coccidioides immitis antigen 2 (Ag2). The JAWS II cells were cotransfected with $\mathrm{pHYG-}$ enhanced green fluorescent protein (EGFP) and pVR1012-C. immitis Ag2 plasmid DNAs using TransIT-TKO reagent. We reproducibly obtained 30\%-50\% transfection efficiency. The transfected cells maintained their immature phenotype and were functionally active. In addition, the flexibility of this agent for expressing multiple antigens (GFP and C. immitis Ag2) offers an advantage of delivering multiple immunogens.

\section{INTRODUCTION}

Dendritic cells are unique antigenpresenting immune cells that play an important role in regulating the host immune responses $(1,2)$. Immature dendritic cells have a unique capability of sensing the maturation signals of infection or inflammation and inducing primary immune responses (3). These cells can internalize and process bacterial, fungal, viral, and other particulate antigens for major histocompatibility complex (MHC) classes I and II antigen presentation to $\mathrm{T}$ cells. Following an encounter with an antigen, dendritic cells mature into antigen-presenting cells, up-regulate their chemokine and co-stimulatory receptors, and migrate to secondary lymphoid organs where they can prime naive $\mathrm{T}$ cells and naive and memory $\mathrm{B}$ cells and activate natural killer cells to induce specific immune responses (4-6). Because of these unique characteristics, dendritic cells have gained considerable interest in the field of vaccine development and immunotherapy. Ex vivo activation and maturation of dendritic cells have been achieved by incubating the has been very difficult, with efficien-

cells with viable (7-11) or killed infectious agents or tumor cells and derived antigens $(11,12)$. It has been noted that genetic transfection is a highly effecdendritic cells with sustained immunomodulatory activity $(13,14)$. However, transfection of dendritic cells with
plasmid DNA using nonviral reagents cies varying from less than $1 \%$ to $20 \%$ (15-17). Adenoviral-mediated transfection, on the other hand, is successful but requires a fairly high multiplicity of infection (100-1000) $(18,19)$, and the utility of this approach is limited by the finding that cellular immune responses are elicited against viral proteins as well $(20,21)$, resulting in mild activation of the phenotype of dendritic cells (22-24). Here we describe an improved transfection protocol, using the nonviral nonliposomal lipid polymer, TransIT-TKO ${ }^{\circledR}$ transfection reagent (Mirus, Madison, WI, USA), for transfecting murine dendritic cells with the gene that encodes Coccidioides immitis antigen $2(\mathrm{Ag} 2)$. Experimental studies in a murine model have established that C. immitis Ag2 DNA, a cell wall glycoprotein, induces protective immunity against lethal challenge (25).

\section{MATERIALS AND METHODS}

In the present study, murine dendritic cells (JAWS II) (ATCC, Manassas, VA, USA) were transfected with pHYG-enhanced green fluorescent protein (EGFP) (BD Biosciences Clontech, Palo Alto, CA, USA) and C. immitis Ag2 cDNA ligated to pVR1012 (Vical, San Diego, CA, USA). The JAWS II dendritic cells were derived from the bone marrow of C57/BL6 mice and express an immature phenotype. The plasmid DNA was prepared using an EndoFree ${ }^{\circledR}$ Plasmid Maxi kit (Qiagen,

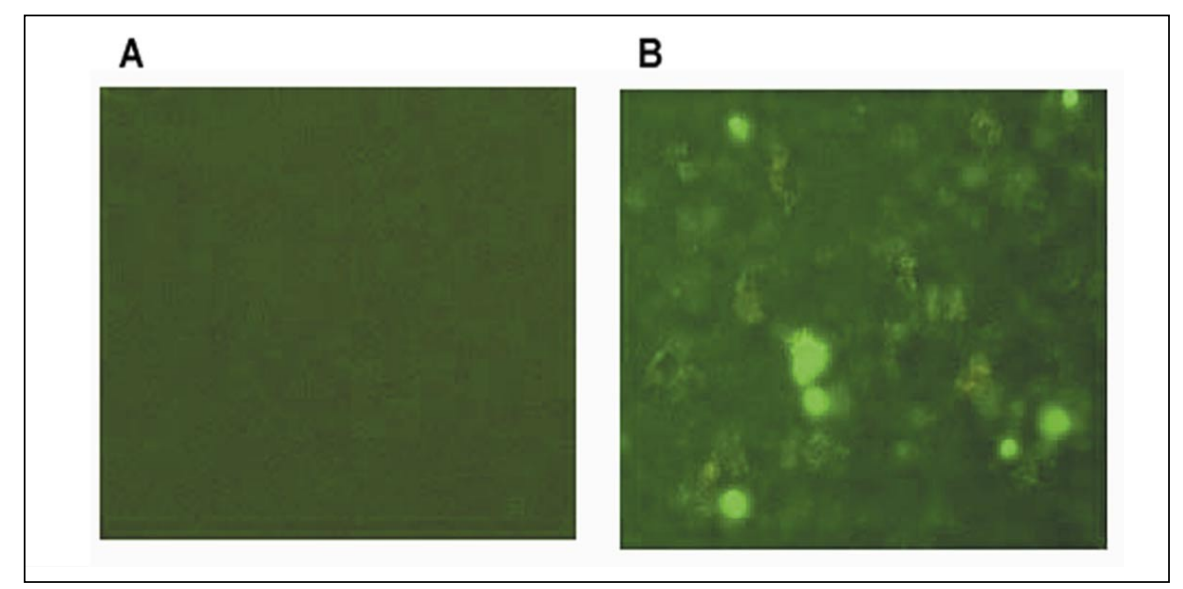

Figure 1. Fluorescent micrographs of (A) nontransfected and (B) pHYG-enhanced green fluorescent protein (EGFP) and pVR1012-Coccidioides immitis antigen 2 (Ag2) plasmid DNA-cotransfected JAWS II cells. The green fluorescence shows expression of GFP. These results are representative of three different experiments. These photographs were taken after a 48-h transfection. 
Valencia, CA, USA). The JAWS II cells were maintained in complete $\alpha$-minimum essential medium containing ribonucleosides and deoxyribonucleosides (Sigma, St. Louis, MO, USA), 20\% fetal bovine serum (Invitrogen, Carlsbad, CA, USA), 4 mM L-glutamine, 5 $\mathrm{ng} / \mathrm{mL}$ recombinant mouse granulocyte macrophage colony-stimulating factor (GM-CSF) (Peprotech, Rocky Hill, NJ, USA), penicillin-streptomycin $(100 \mu \mathrm{g} / \mathrm{mL}$ penicillin and $100 \mathrm{U} / \mathrm{mL}$ streptomycin; Invitrogen), and 50 $\mu \mathrm{g} / \mathrm{mL}$ gentamycin (Invitrogen). The cells were allowed to grow overnight to $50 \%-60 \%$ monolayer confluency in 24-well plates. The cells were washed with serum-free Dulbecco's minimum essential medium (DMEM; Invitrogen) before transfection. The transfection reagent/DNA complex (4 $\mu \mathrm{L}: 2 \mu \mathrm{g}$ each plasmid DNA) was prepared in serum-free DMEM by adding plasmid DNAs $(2 \mu \mathrm{g}$ pHYG-EGFP and 2 $\mu \mathrm{g}$ pVR1012-C. immitis Ag2 plasmid

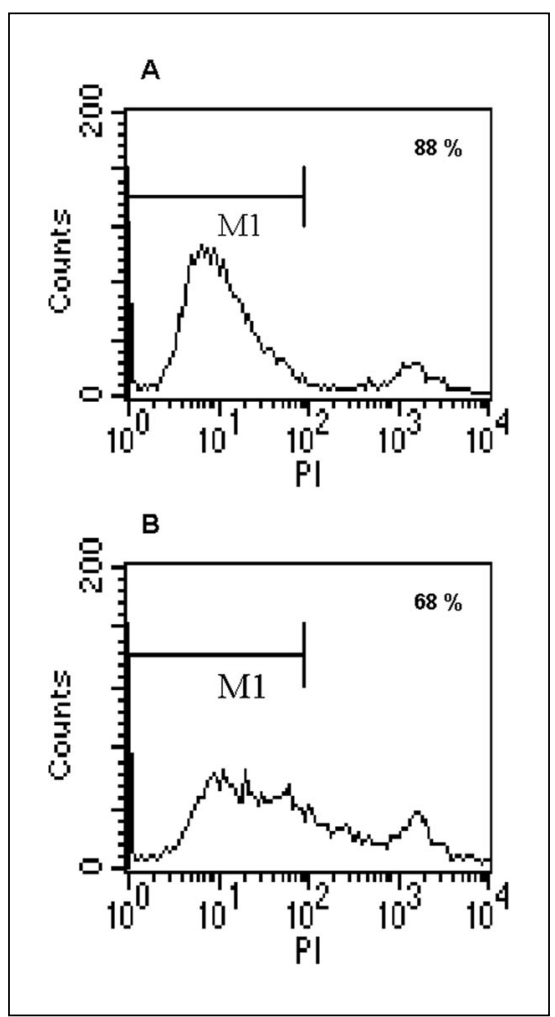

Figure 2. Flow cytometry analysis of (A) propidium iodide-stained nontransfected and $(B)$ pHYG-enhanced green fluorescent protein (EGFP) and pVR1012-Coccidioides immitis antigen 2 (Ag2) plasmid DNA-cotransfected JAWS II cells. Numbers in the upper right indicate the percentage of viable cells.
DNA) to the TransIT-TKO reagent (in $150 \mu$ L DMEM). The mixture was kept at room temperature for at least $5 \mathrm{~min}$ before adding it to the cells. The cells were incubated at $37^{\circ} \mathrm{C}$ in a $5 \% \mathrm{CO}_{2}$ incubator. After a 4-h incubation, an additional $250 \mu \mathrm{L}$ of complete medium were added. The percent transfection efficiency was evaluated by visual enumeration of the green fluorescencetransfected cells versus the total number of cells using a fluorescent microscope with the appropriate filter (Olympus Optical Co. Ltd., Tokyo, Japan). After a 24-h transfection, both the transfected and nontransfected JAWS II cells were stained with propidium iodide to confirm the viability.

The expression of the $C$. immitis $\mathrm{Ag} 2$ protein was detected by dot-blot immunoblotting. Briefly, the cells were washed once with Dulbecco's phosphate-buffered saline (Invitrogen), and the cell lysates were prepared in homogenization buffer containing $1 \%$ Igepal CA-630, 0.1\% sodium dodecyl sulfate, protease inhibitors $(1.1 \mu \mathrm{M}$ leupeptin, $1 \mu \mathrm{M}$ pepstatin, and $0.2 \mathrm{mM}$ phenylmethylsulfonyl fluoride), and 1 mM EDTA (all from Sigma). Various amounts of cell lysate protein were loaded on prewetted nitrocellulose paper (Schleicher \& Schuell, Keene, NH, USA) in Tris-buffered saline (TBS; 20 $\mathrm{mM}$ Tris-HCl, $\mathrm{pH}$ 7.5, $150 \mathrm{mM} \mathrm{NaCl}$ ). The nonspecific sites were blocked by incubating with $5 \%$ bovine serum albumin in TBST (TBS containing $0.05 \%$ Tween ${ }^{\circledR} 20$ ). The nitrocellulose sheet was then incubated with goat anti-Ag2 antibody (1:1000) followed by incubation with secondary alkaline phosphatase-labeled rabbit anti-goat immunoglobulin $\mathrm{G}(\mathrm{IgG})$ antibody (1: 10,000; Sigma). The immunocomplex was detected by an AP Conjugate Substrate kit (Bio-Rad Laboratories, Hercules, CA, USA).

We also confirmed the phenotype and stimulatory capacity of the pVR1012-C. immitis Ag2 plasmid DNA-transfected JAWS II cells. The cells were transfected with pVR1012 or pVR1012-C. immitis Ag2 plasmid DNA according to the method described here using TransIT-TKO reagent. After a 24$\mathrm{h}$ transfection, cells were incubated with Escherichia coli O111:B4 lipopolysaccharide (LPS) (final concentration 100 
$\mathrm{ng} / \mathrm{mL}$; Sigma) for another $24 \mathrm{~h}$. The cells were stained with fluorochromelabeled anti-mouse CD14, CD11c, CD86, MHC class II (IA/E), CD80, and CD40 antibodies, according to the method described by the manufacturer (BD Pharmingen, San Diego, CA, USA), and fluorescence analysis was performed using a FACSCalibur ${ }^{\mathrm{TM}}$ flow cytometer and CellQuest ${ }^{\mathrm{TM}}$ V3.1 software (both from BD Biosciences, San Jose, CA, USA). The mean fluorescent index above background was obtained by using the formula [(geometric mean of fluorescence intensity $\times \%$ gated cells)/100] - background values.

\section{RESULTS}

The transfection efficiency ranged from $30 \%$ to $50 \%$ in three separate experiments using different batch products of TransIT-TKO reagent (Figure 1). Approximately $70 \%$ of the transfected cells remained viable after a 24-h transfection, as compared to $88 \%$ of the nontransfected cells (Figure 2). The pVR1012-C. immitis Ag2 plasmid DNA-transfected JAWS II cells expressed the $C$. immitis $\mathrm{Ag} 2$ protein (Figure 3).

The JAWS II cells maintained their immature phenotype during transfection, and there was little or no change in ex-

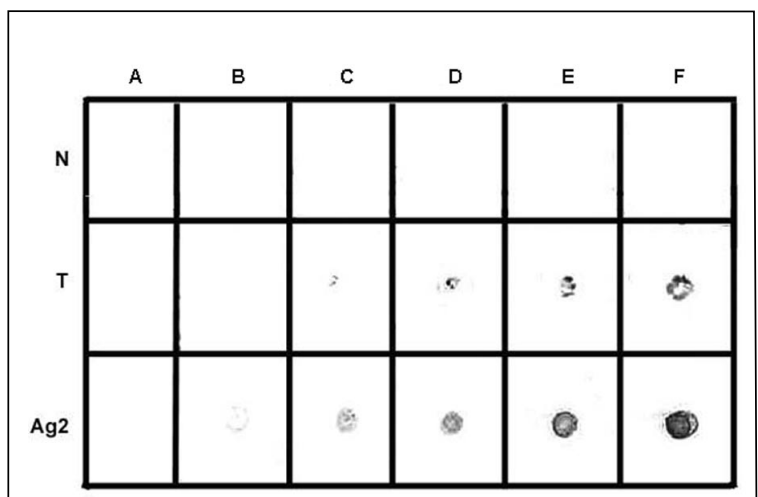

Figure 3. Coccidioides immitis antigen 2 (Ag2) expression by transiently cotransfected JAWS II cells after a 24-h transfection as observed by dot-blot immunoblotting. Lanes A-F: $0,1.75,2.5$, $5.0,10.0$, and $20.0 \mu \mathrm{g}$ total cellular protein, respectively, from nontransfected JAWS II cells (N) and pHYG-enhanced green fluorescent protein (EGFP) and pVR1012-C. immitis Ag2 plasmid DNA-cotransfected JAWS II cells (T). Lanes A-F: 0, 1.0, 2.0, 5.0, 10.0, and $20.0 \mu \mathrm{g}$, respectively, recombinant $C$. immitis $\mathrm{Ag} 2$ protein $(\mathrm{Ag} 2)$. Black dots show the immunocomplex. The results are representative of three different experiments. pression of cell surface markers in either the pVR1012 vector or the pVR1012-C. immitis Ag2 plasmid DNA-transfected JAWS II cells, as compared to the nontransfected JAWS II cells, except for CD80 and CD86 (Figure 4A). However, after LPS treatment, nontransfected and pVR1012- or pVR1012-C. immitis Ag2 plasmid DNA-transfected JAWS II cells and cytokines [tumor necrosis factor $\alpha$ $\mathrm{NF} \alpha$ ), interleukin (IL)-1 $\beta$, and IL-12; data not shown] secretion.

\section{DISCUSSION}

The present results indicate that we can reproducibly obtain improved transfection in dendritic cells using TransIT-TKO nonviral transfection reagent. The transfected JAWS II cells remain viable and functionally active has limitations of being labor-intensive and its utility for gene therapy has imunogenic and safety concerns, the brief mixing of the commercially available transfection reagent and plasmid DNA. Moreover, the flexibility of this agent for expressing multiple antigens (GFP and C. immitis Ag2) offers advantages in delivering multiple immunogens. The transfection protocol reported here can provide a convenient and rapid means of screening novel vaccine candidate genes emerging from genomic and serologic studies for those giving optimal immune responses. C. immitis [a biological safety level-3 (BSL-3) class pathogen] causes pulmonary and extrapulmonary systemic infection in endemic areas of the Southwestern U.S. and Mexico. Treatment of coccidioidomycosis with antifungal agents is difficult, and patients often fail to respond to therapy. Immunotherapeutic in- tervention using transfected dendritic cells could provide an alternative or adjunctive means for reducing the mortality associated with this disease.

\section{ACKNOWLEDGMENTS}

This work was supported by the California HealthCare Foundation, the Department of Health Services of the State of California, and California State University at Bakersfield.

\section{REFERENCES}

1.Banchereau, J. and R.M. Steinman. 1998. Dendritic cells and the control of immunity. Nature 392:245-252.

2.Bell, D., J.W. Young, and J. Banchereau. 1999. Dendritic cells. Adv. Immunol. 72: 255-324.

3.Steinman, R.M. 1991. The dendritic cell system and its role in immunogenicity. Annu. Rev. Immunol. 9:271-296.

4.Ingulli, E., A. Mondino, A. Khoruts, and M.K. Jenkins. 1997. In vivo detection of dendritic cell antigen presentation to CD4+ T cells. J. Exp. Med. 185:2133-2141.

5.Wykes, M., A. Pombo, C. Jenkins, and G.G. MacPherson. 1998. Dendritic cells interact directly with naïve B lymphocytes to transfer antigen and initiate class switching in a primary T-dependent response. J. Immunol. 161: 1313-1319.

6.Fernandez, N.C., A. Lozier, C. Flament, P. Ricciardi-Castagnoli, D. Bellet, M. Suter, M. Perricaudet, T. Tursz, et al. 1999. Dendritic cells directly trigger NK cell functions: cross-talk relevant in innate anti-tumor immune responses in vivo. Nat. Med. 5: 405-411.

7.Hickman, S.P., J. Chan, and P. Salgame. 2002. Mycobacterium tuberculosis induces differential cytokine production from dendritic cells and macrophages with divergent effects on naïve $\mathrm{T}$ cell polarization. J. Immunol. 168:4636-4642.

8.Tascon, R.E., C.S. Soares, S. Ragno, E. Stavropoulos, E.M. Hirst, and M.J. Colston. 2000. Mycobacterium tuberculosis-activated dendritic cells induce protective immunity in mice. Immunology 99:473-480.

9.Henderson, R.A., S.C. Watkins, and J.L. Flynn. 1997. Activation of human dendritic cells following infection with Mycobacterium tuberculosis. J. Immunol. 159:635-643.

10.Ojcius, D.M., Y. Bravo de Alba, J.M. Kanellopoulos, R.A. Hawkins, K.A. Kelly, R.G. Rank, and A. Dautry-Varsat. 1998. Internalization of Chlamydia by dendritic cells and stimulation of Chlamydia-specific T cells. J. Immunol. 160:1297-1303.

11.Baourguin, I., M. Mosser, D. Buzoni-Gatel, F. Tielemans, D. Bout, J. Urbain, and $O$. Leo. 1998. Murine dendritic cells pulsed in vitro with Toxoplasma gondii antigens induce protective immunity in vivo. Infect. Immun. 

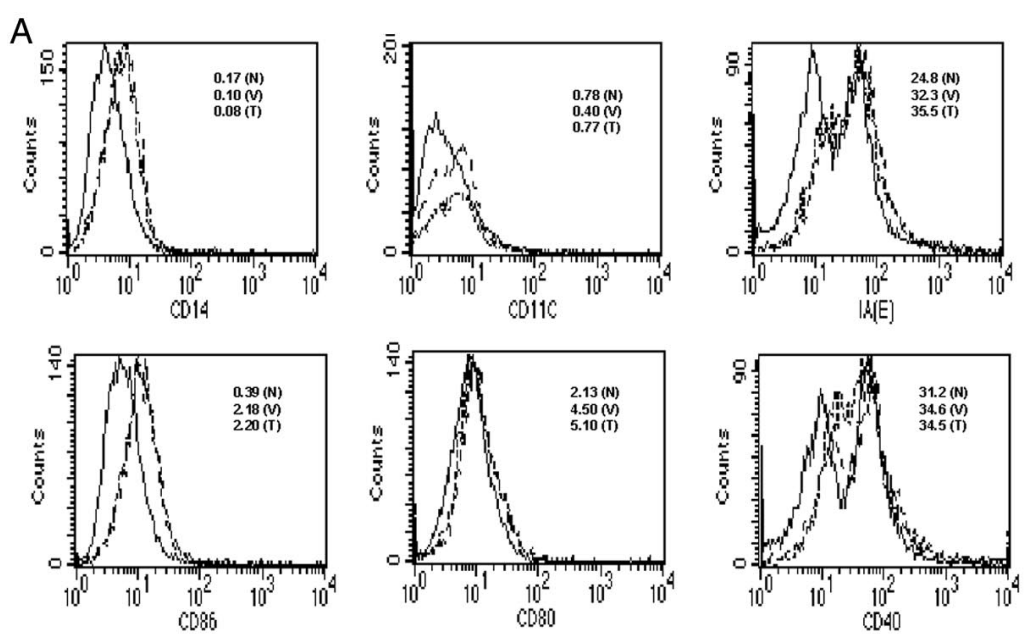

Nontransfected $(\mathrm{N})$,

pVR1012 transfected $(V)$

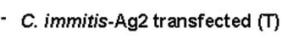

B

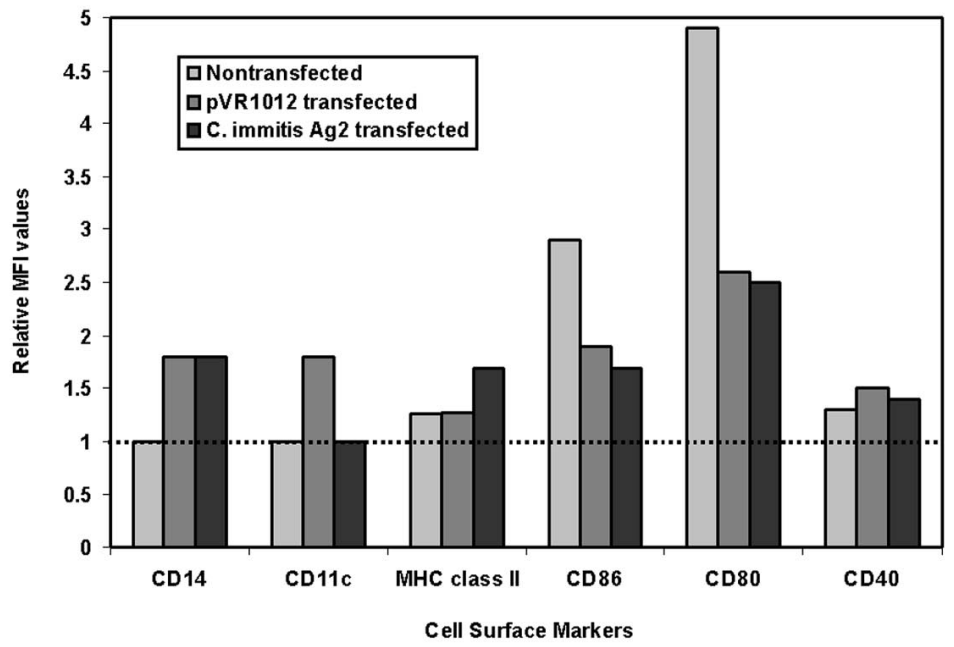

Figure 4. Comparison of cell surface marker expression in nontransfected, pVR1012-transfected, and pVR1012-Coccidioides immitis antigen 2 (Ag2) plasmid DNA-transfected JAWS II cells. (A) Cell surface expression of CD14, CD11c, major histocompatibility complex (MHC) class II (IA/E), CD86, CD80, and CD40 in nontransfected (N), pVR1012 vector-transfected (V), or pVR1012-C. immitis Ag2 plasmid DNA-transfected (T) JAWS II cells. The numbers in the upper right indicate the mean fluorescent index (MFI) above background. (B) Cell surface expression of CD14, CD11c, MHC class II (IA/E), CD86, CD80, and CD40 in nontransfected, pVR1012 vector plasmid-transfected, or pVR1012C. immitis Ag2 plasmid DNA-transfected JAWS II cells in response to Escherichia coli lipopolysaccharide (LPS) $(100 \mathrm{ng} / \mathrm{mL})$. The MFI values for cell surface markers presented here on the y-axis show the ratio of MFI values in respective (LPS-treated to untreated) cells. The horizontal dashed line at MFI value 1 represents the MFI in untreated cells. The results are from one representative experiment performed in triplicate.

66:4867-4874.

12.Richards, J.O., N.M. Ampel, J.N. Galgiani, and D.F. Lake. 2001. Dendritic cells pulsed with Coccidioides immitis lysate induce antigen-specific naïve $\mathrm{T}$ cell activation. J. Infect. Dis. 184:1220-1224.

13.Cella, M., M. Salio, Y. Sakakibara, H. Langen, I. Julkunen, and A. Lanzavecchia. 1999. Maturation, activation, and protection of dendritic cells induced by double-stranded RNA. 189:821-829.

14.Song, W., H.L. Kong, H. Carpenter, H.
Torii, R. Granstein, S. Rafii, M.A. Moore, and R.G. Crystal. 1997. Dendritic cells genetically modified with an adenovirus vector encoding the cDNA for a model antigen induce protective and therapeutic antitumor immunity. J. Exp. Med. 186:1247-1256.

15.Van Tendeloo, V.F., H.W. Snoeck, F. Lardon, G.L. Vanham, G. Nijs, M. Lenjou, L. Hendriks, C. Van Broeckhoven, et al. 1998. Nonviral transfection of distinct types of human dendritic cells: high-efficiency gene transfer by electroporation in to hematopoi- etic progenitor- but not monocyte-derived dendritic cells. Gene Ther. 5:700-707.

16.Lohmann, S., K. Galle, J. Konp, and A.H. Enk. 2000. CD83+ human dendritic cells transfected with tumor peptide cDNA by electroporation induce specific T-cell responses: a potential tool for gene immunotherapy. Cancer Gene Ther. 7:605-614.

17.Irvine, A.S., P.K.E. Trinder, D.L. Laughton, H. Ketteringham, R.H. Mc Dermott, S.C.H. Reid, A.M.R. Haines, A. Amir, et al. 2000. Efficient nonviral transfection of dendritic cells and their use for in vivo immunization. Nat. Biotechnol. 18:1273-1278.

18.Arthur, J.F., L.H. Butterfield, M.D. Roth, L.A. Bui, S.M. Kiertscher, R. Lau, S. Dubinett, J. Glaspy, et al. 1997. A comparison of gene transfer methods in human dendritic cells. Cancer Gene Ther. 4:17-25.

19.Korst, R.J., A. Mahtabifard, R. Yamada, and R.G. Crystal. 2002. Effect of adenovirus gene transfer vectors on the immunologic functions of mouse dendritic cells. Mol. Ther. 5:307-315.

20.Jooss, K., H.C.J. Ertl, and J.M. Wilson. 1998. Cytotoxic T-lymphocyte target proteins and their major histocompatibility complex class I restriction in response to adenovirus vectors delivered to mouse liver. J. Virol. 72: 2945-2954.

21.Yang, Y., K.U. Jooss, O. Su, H.C.J. Ertl, and J.M. Wilson. 1996. Immune responses to viral antigens versus transgene product in the elimination of recombinant adenovirusinfected hepatocytes in vivo. Gene Ther. 3: 137-144.

22.Hirschowitz, E.A., J.D. Weaver, G.E. Hidalgo, and D.E. Doherty. 2000. Murine dendritic cells infected with adenovirus vectors show signs of activation. Gene Ther. 7: 1112-1120.

23.Morelli, A.E., A.T. Larregina, R.W. Ganster, A.F. Zahorchak, J.M. Plowey, T. Takayama, A.J. Logar, P.D. Robbins, et al. 2000. Recombinant adenovirus induces maturation of dendritic cells via an NK- $\kappa B$-dependent pathway. J. Virol. 74:9617-9628.

24.Jonuleit, H., T. Tuting, J. Steitz, J. Bruck, A. Giesecke, K. Steinbrink, J. Knop, and A.H. Enk. 2000. Efficient transduction of mature CD83+ dendritic cells using recombinant adenovirus suppressed $\mathrm{T}$ cell stimulatory capacity. Gene Ther. 7:249-254.

25.Magee, D.M. and R.A. Cox. 1996. Interleukin-12 regulation of host defenses against Coccidioides immitis. Infect. Immun. 64: 3609-3613.

Received 25 February 2003; accepted 4 June 2003.

Address correspondence to Shanjana Awasthi, Department of Pathology, University of Texas Health Science Center at San Antonio, 7703 Floyd Curl Drive, San Antonio, TX 78229, USA. e-mail: awasthis@uthscsa.edu 